\title{
Classic Ronchi test and its variants for quality control of various optical surfaces
}

\author{
A.S. Malenko, V.N. Borovytsky \\ National Technical University of Ukraine "Kyiv Polytechnic Institute" \\ Optical Engineering Department, 37, prospect Peremogy, 03056 Kyiv, Ukraine \\ Phone: +38-044-454-9477; e-mail: omalenko@ukr.net; WEB: ooep.kpi.ua
}

\begin{abstract}
Performed in this work is the analysis of the optical Ronchi interferometer circuit and its upgrading to test quality of various optical surfaces. Briefly described in this paper is the classic test by Ronchi, shown is every upgraded circuit diagram of the interferometer and their principle of operation. Also, it is demonstrated interferential patterns for each method allowing one to determine which aberrations are present in the tested optics. With this method, when one can only visually detect aberrations, it seems to be not accurate. But with digital image processing the interferential pattern, special mathematical models and algorithms, aberrations that are present in the optical surface can be calculated with very high accuracy. Therefore, the methods of control offered in this paper provide fast and accurate results for the data circuits to be simply assembled and configured.
\end{abstract}

Keywords: Ronchi test, interferential pattern, aberration.

Manuscript received 08.04.16; revised version received 13.07.16; accepted for publication 13.09.16; published online 04.10.16.

\section{Introduction}

Nowadays, industry produces many different optical components. The quality of the production of these parts is determined using various methods of control. For the manufacture of modern high-precision optical devices such as settings for photolithography, laser systems, optical telescopes and microscopes, and so on, one needs to control quality of the surface to within units and even fractions of nanometer. The area controlled surface may be a few square millimeters. For this kind of measurements, they use the interferential methods. Usually, these methods are based on schemes of Michelson, Mach-Zehnder, Jamin and Fizeau interferometers. Also, there is non-interferential testing method, such as sensor by Shaka-Hartman. But all these schemes have major shortcomings, such as the complexity of their construction, adjustment of mirrors, optical components that make the interferometer to be very expensive. Therefore, the Italian scientist Ronchi proposed a new, fast method for evaluating wavefront interferometric scheme of the lateral shear interferometer. This Ronchi method is used for rapid detection of wave aberrations with time for the largest spherical mirror lens in Arcetri astronomical observatory. Currently, a very promising installation based on the Ronchi scheme is built, which will control quality lenses of smartphone. Because every year smartphones become thinner, and lens design becomes more complex and increasingly made. 
In this paper, we consider the basic modernization of the classical Ronchi test. Currently, the Ronchi test allows one to control the wave aberration of spherical, parabolic mirrors, spherical and aspherical lenses. Used for accurate and fast calculation of aberrations are digital image processing and special mathematical models and algorithms for the resulting interferential pattern in the control of the optical surface.

\section{Classic Ronchi test}

For many years the classic test is used to detect the wave aberrations of spherical mirrors. The scheme of this method is shown in Fig. 1.

The principle of the scheme operation is as follows: radiation from a point source (1) reaches the tested mirror (2) and is reflected from it. The reflected spherical wave passes through the diffraction grating (3) that divides it into several identical waves (diffraction maxima) displaced by a certain amount. The next element is a slot diaphragm (4), which separates only the central $m_{0}$, first $m_{1}$ and minus first $m_{-1}$ maximum. Further, these peaks overlap and form an interferential pattern that can be seen on the screen (5) (Fig. 2).

If the tested mirror (2) is made thoroughly and without defects, on the screen (5) one can observe the interferential pattern, in which the stripes are of the same width and parallel to each other. But if the tested mirror (2) is manufactured with defects, the interferential stripes are curved. Fig. 3a shows the interferential pattern, when aberration is absent, and Fig. $3 \mathrm{~b}$ - when aberration is available.

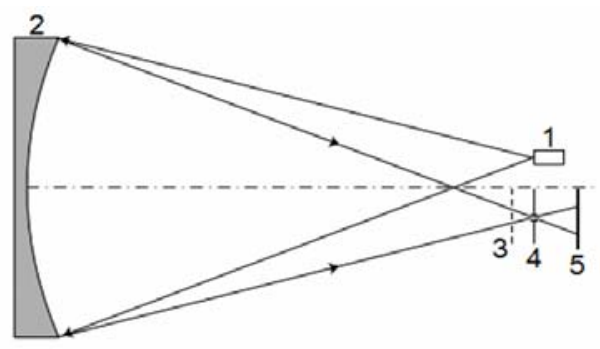

Fig. 1. Ronchi interferometer scheme to control the wave aberrations of spherical mirrors: 1 - point source of radiation 2 - tested mirror, 3 - diffraction grating, 4 - slot diaphragm, 5 - the screen on which we see the interferential pattern.

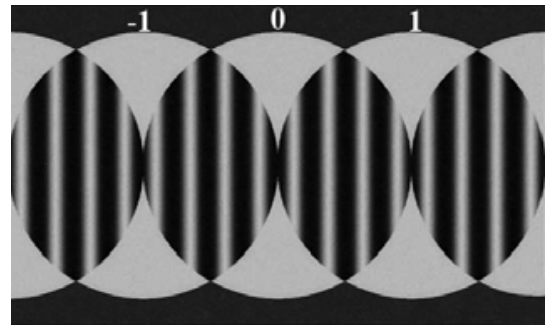

Fig. 2. Overlaping diffraction peaks form an interferential pattern.

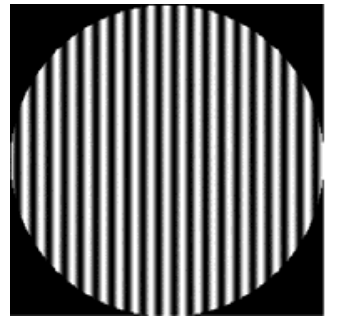

a)

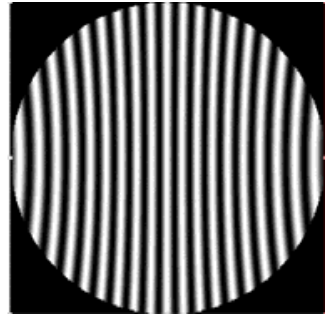

b)
Fig. 3. The interferential patterns: a) no aberration, b) with spherical aberration.

\section{Ronchi test for parabolic mirrors}

Used in this test method for parabolic mirrors are special substructured diffraction gratings. The substructured diffraction grating is a grating formed in such a manner that one adds two or more gratings with different spatial frequencies. Fig. 4 shows the receipt for making such lattice.

A simplified diagram of the setup is shown in Fig. 5. The classical Ronchi scheme was taken as a base but slightly modernized. The substructured grating was displayed using the LC display, and the interferential pattern was created in the plane of CCD matrix. To calculate the wave aberration, the authors of this method developed a special mathematical model and algorithm for processing the digital image of the interferential pattern. As a result, they obtained numeric values of aberrations. The substructured grating was generated using a special software and then displayed on the LC display.

The principle of this scheme is similar to the classic Ronchi test, but instead of a diffraction grating we used LC display and built the interferential pattern in the plane of the CCD matrix in order to be able to provide digital image processing of the picture. Fig. 6 shows the interferential patterns obtained using this method. It should be noted that this method increases the spatial resolution of gratings and provide testing both hyperbolic and spherical surfaces.
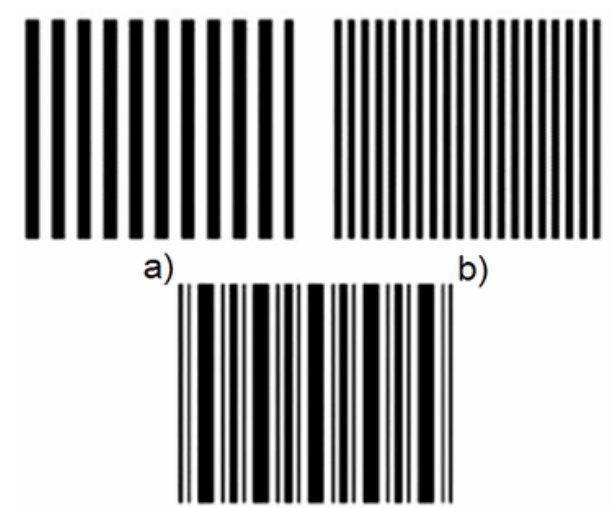

c)

Fig. 4. (a) and (b) - ordinary diffraction gratings with different spatial frequencies; (c) - substructured grating obtained by combination of the gratings (a) and (b). 


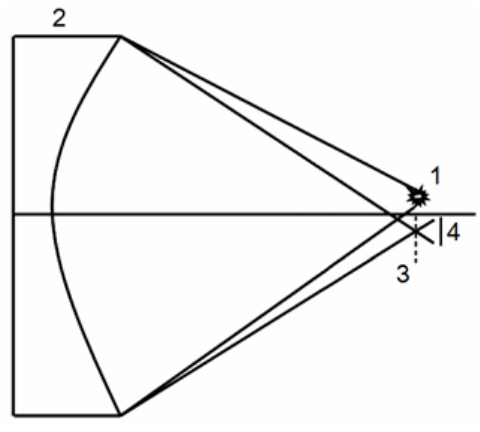

Fig. 5. Ronchi scheme for testing the parabolic mirrors. 1 point source of radiation, 2 - tested mirror, 3 - LC display that shows the substructured grating, 4 - CCD matrix.

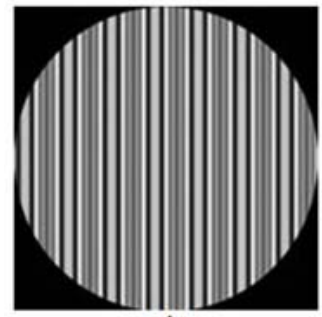

a)

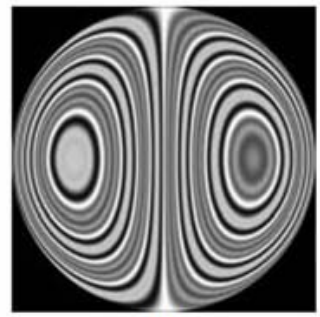

b)
Fig. 6. The interferential patterns: a) without aberrations, b) with spherical aberration.

\section{Ronchi test for spherical lenses}

This method is very similar to the test method for spherical mirrors. Shown in Fig. 7 is the Ronchi scheme for this method.

How it works. After leaving the collimator $(1-4)$, the flat wavefront reaches the objective (5). This lens (5) turns the flat wavefront to spherical. Then this front passes through the diffraction grating (6). This grating separates the spherical wavefront and forms several simple fronts shifted to one another. Then the slot diaphragm (7) allows transmitting only two wavefronts. When they overlap, there formed is the interferential pattern that can be seen on the screen (8). If the lens (6) forms an ideal spherical wavefront, the interferential fringes are parallel, and if not, then the stripes will be distorted.

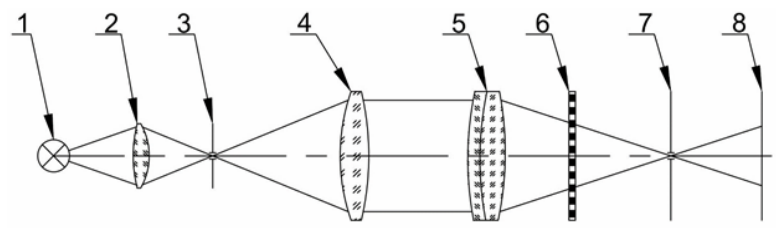

Fig. 7. Ronchi interferometer for testing thin spherical lenses. 1 - radiation source, 2 - condenser, 3 - point diaphragm, 4 collimator, 5 - tested optical component, 6 - diffraction grating, 7 - slot diaphragm, 8 - screen.
The obtained interferential patterns are the same as in Fig. 3. To calculate the aberrations of the third and fifth orders, the polynomial Zernike function with a limited number of terms is usually applied:

$$
\begin{aligned}
& W(\rho, \varphi)=W_{11}(\rho, \varphi)+W_{20}(\rho, \varphi)+ \\
& +W_{22}(\rho, \varphi)+W_{31}(\rho, \varphi)+W_{40}(\rho, \varphi)+ \\
& W_{42}(\rho, \varphi)+W_{51}(\rho, \varphi)+W_{60}(\rho, \varphi) .
\end{aligned}
$$
follows:

The functions of each aberration term are as

$W_{11}(\rho, \varphi)=C_{11} \rho \cos \varphi-$ distortion;

$W_{20}(\rho, \varphi)=C_{11}\left(2 \rho^{2}-1\right)-$ defocus;

$W_{22}(\rho, \varphi)=C_{22} \rho^{2} \cos 2 \varphi-$ astigmatism of the third order;

$W_{31}(\rho, \varphi)=C_{31}\left(3 \rho^{3}-2 \rho\right)-$ third order coma;

$W_{40}(\rho, \varphi)=C_{40}\left(6 \rho^{4}-6 \rho^{2}+1\right)-$ the spherical aberration of the third order;

$W_{42}(\rho, \varphi)=C_{42}\left(4 \rho^{4}-3 \rho^{2}\right)-$ fifth order astigmatism;

$W_{51}(\rho, \varphi)=C_{51}\left(10 \rho^{5}-12 \rho^{3}+3 \rho\right)-$ fifth order coma;

$W_{60}(\rho, \varphi)=C_{60}\left(20 \rho^{6}-30 \rho^{4}+12 \rho^{2}-1\right)-$ spherical aberration of fifth order;

where $\rho$ and $\varphi$ are polar coordinates that are related to Cartesian coordinates in the following way: $x=\rho \cos \varphi$, $y=\rho \sin \varphi, C_{n m}-$ the coefficient of aberration.

\section{Ronchi test for plane aspherical lenses}

Currently, interest to aspherical lenses grows, because these lenses eliminate spherical aberration and reduce other aberrations inherent to spherical lenses, since their radius varies with distance from the optical axis. Also, aspherical lenses can help to simplify optical system design by minimizing the number of elements. In addition, they can produce sharper images than conventional lenses. As a result, aspherical lenses provide better image quality, compactness and easeness of the optical system. It is clear that the quality of the lens should be controlled, and nowadays - very quickly. Therefore, for quick aspherical lens control the designed scheme was based on the classic Ronchi test. The optical scheme for this test is shown in Fig. 8.

The operation principle of the scheme: radiation source (1), micro-lens (2), point diaphragm (3) and collimator (4) form a parallel beam. To reduce the intensity of radiation on CCD matrix (8) there used are polarizers (5). The special diffraction gratings (6) were printed on film and inserted between the polarizers. Then, this radiation from the optical system falls on the tested aspherical lens. The interferential pattern is recorded by the CCD matrix. To process this picture, we developed a mathematical model. In Fig. 9, the resulting images are shown for the experiments with different diffraction gratings.

This method can be also used for testing spherical lenses. 


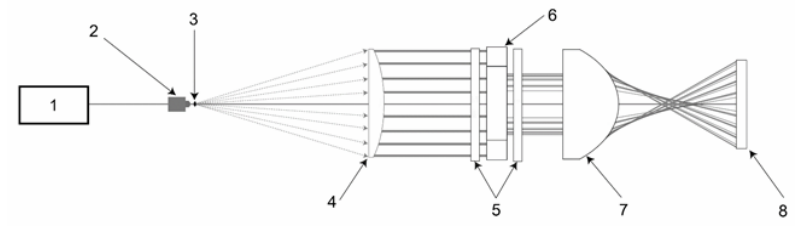

Fig. 8. Scheme of the experimental setup for testing plane aspherical lenses. 1 - radiation source, 2 - micro-lens, 3 point diaphragm, 4 - collimator, 5 - polarizers, 6 - SLM with null screen, 7 - tested aspherical lens, 8 - CCD matrix.
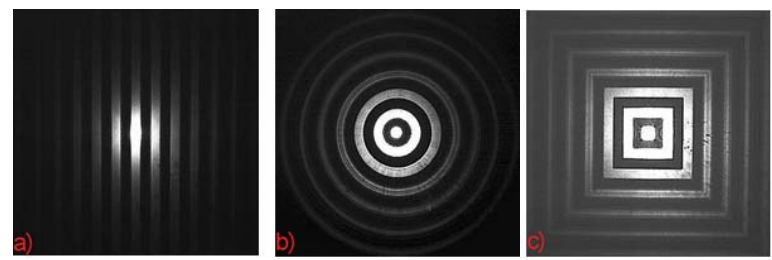

Fig. 9. Images obtained in the course of testing the aspherical lenses with different diffraction gratings: a) linear, b) circular, c) square.

\section{Conclusions}

In this paper, we analyzed a lot of material and briefly described methods by which one can control quality of various optical surfaces (spherical, parabolic, hyperbolic mirror as well as spherical and aspherical lenses). All these methods are based on the classic Ronchi test and modernized for each type of optical surfaces. Using these methods, one can quickly and accurately evaluate various lenses and image quality that they provide. All these methods are modern and some of them use special software for generating diffraction gratings that are then displayed on the LC display. Also, we used a specialized software for digital image processing the received interferential patterns. It enables to numerically calculate aberrations that are present in the tested surfaces. The disadvantage of these methods is that one needs to make two interferential patterns perpendicular to each other. This is done so that one can compare and calculate astigmatism of the third and fifth orders. But this problem is also solved: Makalar proposed a mathematical model when one only needs the only digital image of the interferential pattern. In general, the Ronchi interferometer is simple in assemblage, adjustment, resistant to vibrations, and its components are relatively cheaper than those in other interferometers, where used is a very expensive translucent mirror.

\section{References}

1. Diana Castán Ricaño and Maximino AvendañoAlejo, Designing null screens type sub-structured Ronchi to test a fast plano-convex aspheric lens // Proc. SPIE, 9195, Optical System Alignment, Tolerancing, and Verification VIII, 919509 (September 8, 2014); doi:10.1117/12.2062454.
2. Qingkui Xi, Lei Chen, Rihong Zhu, Yunchen Li and Xueliang Bai, Fast Ronchi test base on a liquid crystal display // Proc. SPIE, 5638, Optical Design and Testing II, 682 (April 06, 2005); doi:10.1117/12.574093.

3. D. Aguirre-Aguirre, F.S. Granados-Agustín and A. Cornejo-Rodríguez, Obtaining the wavefront in the Ronchi test using only one Ronchigram with random coefficients of aberration // Proc. SPIE, 8011, 22nd Congress of the International Commission for Optics: Light for the Development of the World, 801117 (October 25, 2011); doi:10.1117/12.902606.

4. Noel I. Toto Arellano, Gustavo Rodriguez Zurita and Alejandro Cornejo Rodriguez, Physical theory of ronchigrams resulting from unequal strip-widths rulings // Proc. SPIE, 5776, Eighth International Symposium on Laser Metrology, 561 (February 14, 2005); doi:10.1117/12.611857.

5. Juan Manuel Nuñez, Isbak Valdivieso, Javier Salinas-Luna, Adriana Nava-Vega and Joel H. Castro-Chacón, Relationship between the fnumber and the ruling frequency for a better discrimination of optical surfaces with the classical Ronchi test // J. Astron. Telesc. Instrum. Syst. 1(3), 034004 (Aug 27, 2015).

6. Yaoltzin Luna-Zayas, Fermín-Granados Agustín and Alejandro Cornejo-Rodríguez, Ronchi test with sub-structured gratings // Proc. SPIE, 6034, ICO20: Optical Design and Fabrication, 60341W (January 20, 2006); doi:10.1117/12.668170.

7. Javier Salinas-Luna, Fermín Granados-Agustín, Alejandro Cornejo-Rodríguez, Esteban Luna, Juan Jaime Sánchez-Escobar et al., Ronchi test with variable-frequency rulings // Opt. Eng. 48(1), 013604 (January 29, 2009).

8. Miguel Mora-Gonza'lez and Noe Alcala' Ochoa, Sinusoidal liquid crystal display grating in the Ronchi test // Opt. Eng. 42(6), p. 1725-1729 (June 01, 2003).

9. Daniel Aguirre-Aguirre, Brenda VillalobosMendoza, Fermín S. Granados-Agustín, Rafael Izazaga-Pérez, Manuel Campos-García, et al., Substructured Ronchi gratings from the linear combination of classical gratings // Opt. Eng. 53(11), 114111 (Nov 21, 2014).

10. Joseph Braat and Augustus J. E. M. Janssen, Improved Ronchi test with extended source // Optical Society of America [S0740-3232(99)00501-3], (September 3, 1998), doi: 10.1364/JOSAA.16.000131.

11. Sukmock Lee and Manuel Guizar-Sicairos, Validation of quantitative Ronchi test through numerical propagation // Optical Society of America (2010), doi: 10.1364/OE.18.018525.

12. V. Ronchi, Le Frange di Combinazioni Nello Studio delle Superficie e dei Sistemi Ottici // Riv. Ottica Mecc. Precis. 2, 9 (1923).

13. V. Ronchi, Due Nuovi Metodi per lo Studio delle Superficie e dei Sistemi Ottici // Ann. Sc. Norm. Super. Pisa, 15 (1923). 